\title{
Troubleshooting an Intrusion Detection Dataset: the CICIDS2017 Case Study
}

\author{
Anonymous author(s)
}

\begin{abstract}
Numerous research studies have demonstrated the effectiveness of machine learning techniques in application to network intrusion detection. And yet, the adoption of machine learning for securing large-scale network environments remains limited. The community acknowledges that network security presents unique challenges for machine learning, and the lack of training data representative of modern traffic remains one of the most intractable issues. New attempts are continuously made to develop high quality benchmark datasets and proper data collection methodologies. The CICIDS2017 dataset is one of the recent results, created to meet the demanding criterion of representativeness for network intrusion detection. In this paper, we revisit CICIDS2017 and its data collection pipeline and analyze correctness, relevance and overall utility of the dataset for the learning task. In the process, we uncover a series of traffic generation, flow extraction and labeling problems that severely affect the validity of data. To counter these issues, we investigate and eliminate their causes and regenerate the dataset. As a result, more than $20 \%$ of traffic traces are reconstructed or relabeled, and machine learning benchmarks demonstrate significant improvements. Our study exemplifies how data collection issues may have enormous impact on model evaluation and provides recommendations for their anticipation and prevention.
\end{abstract}

Index Terms-network intrusion detection, machine learning, benchmark dataset, data collection.

\section{INTRODUCTION}

Network intrusion detection systems (NIDS) are security tools strategically deployed in a network environment that monitor and interpret internal and external traffic in search for malicious activities. Advances in Machine Learning (ML) over the last decades have enabled NIDS to evolve from simple rule-based systems to intelligent automated decision-making engines powered by modern learning algorithms [1]. Despite significant progress in ML-based defensive research, a lot of challenges are still open when it comes to NIDS solutions. For any data-driven technique, the role of high quality, representative datasets cannot be underestimated; this is especially true for NIDS research that has to account for high non-stationarity and strong heterogeneity of network traffic, together with an uncontrollable production environment.

In NIDS research, evaluation on benchmark datasets primarily serves a two-fold purpose: (i) real-world performance estimation of a particular algorithm, and (ii) consistent comparison between different approaches. In this respect, quality of data has a decisive influence on valid outcomes of both objectives. Research has shown that many benchmark datasets do not adequately represent the real problem of network intrusion detection, discrediting performance numbers achieved in laboratory conditions [1]. As a response, over the last 10 years the community has collectively devised the criteria that reliable research traffic data should meet [2]-[4]. These criteria can be seen as objectives of proper dataset generation, be that for capturing real traffic or simulating attack scenarios.

Recently, the Canadian Institute of Cybersecurity released a new dataset - CICIDS2017 [5] - that was meant to address some of the issues that plagued older datasets. CICIDS2017 is comprised of real traffic generated through simulated interactions between hosts in a controlled infrastructure, which means that the resulting network traffic retains basic limitations of a simulated environment. Nevertheless, the authors do a great effort to achieve realism, heterogeneity, diversity and completeness of the dataset in order to maximize its utility for research purposes. Owing to that promise, CICIDS2017 is growing in usage in NIDS research [6]-[9]. Crucially, however, the dataset is largely taken at its face value, assuming that a modern dataset collected with the quality requirements in mind indeed satisfies these criteria. As a result, the studies use successful ML evaluations as a sole confirmation of correctness of CICIDS2017, but do not explicitly validate these results by looking at data. The lack of dedicated analysis sheds uncertainty on the empirical and theoretical research results obtained when leveraging the dataset. We aim to revisit the properties of CICIDS2017 as a NIDS dataset and study to what extent it satisfies the declared requirements.

In this work, we perform an in-depth analysis of the CICIDS2017 dataset and uncover a series of previously unknown issues that may have far-reaching implications on the research activities utilizing the dataset. Namely, we carefully analyze the influential stages of the data collection pipeline - attack simulation, flow construction, labeling, feature extraction and ML benchmarking - and report any observed inconsistencies or oversights. Furthermore, we bring attention to specific design choices made during data collection that could impact experimental results and research conclusions. Equipped with these observations, we regenerate a new version of the dataset and analyze it from a perspective of its utility for the intended learning task.

Our contributions can be summarized as follows:

- We perform a thorough methodological manual analysis of the raw CICIDS2017 dataset and its respective feature extraction tool in order to review the dataset creation pipeline. Our investigation reveals errors in attack simulation, feature extraction, labeling and benchmarking of the dataset. Moreover, we find out that more than $25 \%$ of all flows in the dataset turn out to be meaningless artefacts carrying no real identifying characteristics. For 
several attack categories, their fraction reaches $50 \%$, which is significantly beyond an acceptable noise level. Our investigation confirms that the issue is still present in the newest version of the dataset - CIC-CSE-DS2018.

- We study the negative impact of the dataset errors, artefacts and mislabeled traces on ML algorithms, and provide insights into why these issues can easily pass undetected when using the dataset for NIDS research.

- Building on these findings, we modify the feature extractor and regenerate and relabel the CICIDS2017 dataset based on the original PCAPs. Improved correctness and reliability of the regenerated dataset are further supported by ML benchmarks that reach the highest performance ever reported on these data.

\section{DATASET \& Related WoRK}

CICIDS2017 was created as a response to the dataset quality evaluation criteria composed in earlier studies meant to eliminate common mistakes of other synthetically created datasets [2]-[4]. Specifically, a dataset is considered reliable when it covers the following properties. It should contain (a) real and (b) valid network traffic, such that it constitutes complete scenarios that can be observed in actual production environments. The benign and malicious traces should be (c) correctly labeled, (d) highly variant/diverse in terms of represented services, client behaviours and attacks, (e) correctly implemented, according to commonly accepted standards, and (f) easily updatable, when new services and attacks are introduced. The dataset should also be $(\mathrm{g})$ reproducible, enabling comparison across different datasets, and (h) shareable, containing no private information. Finally, a good dataset should be supplied with (i) documentation and metadata of data collection, including details about the network infrastructure and simulated attack scenarios. CICIDS2017 is asserted to satisfy all of these criteria by design.

The dataset is generated in a complete network topology with diverse devices and operating systems as a testbed, where separate victim and attacker networks communicate over the Internet. To collect benign traffic that models realistic user behaviour, the authors use profiling agents [10] that are trained beforehand on network events generated through genuine human interactions in the network. Communication covers all common available protocols, such as HTTP, HTTPS, FTP, $\mathrm{SSH}$ and email protocols. In order to fulfil the requirement for attack diversity, the authors use the 2016 McAfee report to compose the list of most common attacks: Brute Force attacks, Heartbleed attack, Botnet communication, several variants of DoS attacks, DDoS, Infiltration attack and Web attacks. In total, the dataset comprises over 2.8 million traffic traces categorized into 15 classes. Individual traffic traces are represented by a feature vector of 80 extracted high-level statistical features, manually engineered based on expert knowledge of traffic characteristics relevant to intrusion detection.

Since 2017, plenty of studies have deployed CICIDS2017 (and its latest version CSE-CIC-IDS2018) for NIDS research [6]-[9]. The studied research problems span ML- assisted network intrusion detection, novelty detection, anomaly detection, online detection, with a large variety of learning algorithms and traffic representations - raw PCAPs, statistical features, graphs, etc. Recently, both datasets started being considered for adversarial machine learning research, such as evasion of botnet detection [11]. The conclusions reached by these studies largely rely on correctness and validity of the dataset in all its forms. There are studies that have analyzed features used in the dataset [12] and raised the issue of class imbalance [13]. However, their analysis does not address correctness and validity of the dataset.

\section{MANUAL ANALYSIS AND CORRECTIONS}

We select CICIDS2017 for in-depth analysis based not only on its growing adoption on the literature, but also on sufficient transparency and reproducibility of its generation process. ${ }^{1}$ This section describes the main analysis of the dataset and its feature extraction tool. Our goal here is to revise the aforementioned properties of a good NIDS dataset, which we do through examining the following dataset creation stages:

- Attack simulation. Most research datasets for NIDS contain malicious traces representative of target malicious activities. To include a wide variety of attacks, and save the effort of annotating real traffic, the attacks are often simulated with open source tools or custom scripts.

- Flow construction. Raw network traffic is a continuous data stream that needs to be monitored and analyzed in real time. It is therefore necessary to decide what the level of granularity will be, i.e. what constitutes a single input unit for the detection system. Traffic is commonly processed at granularity of a flow associated with one complete network connection.

- Labeling. When working with a supervised detection system, the aim is not only to distinguish between benign and malicious traffic, but to label malicious traffic according to the attack class that it belongs to. When training a Machine Learning based classifier for this purpose, it needs to be supplied with example network data from all classes that it needs to be able to detect, along with the correct label for each input. When testing the model, comparing correct labels with predicted classes allows to assess the model's performance.

- Feature extraction. Traditional machine learning systems cannot operate on multidimensional raw traffic data, and instead require some form of structured input. Therefore, a flow-based intrusion detection system would convert each raw traffic flow to a fixed-length structured vector that encapsulates all the important information about the flow in a form of numerical and/or categorical features. For instance, a NIDS could extract statistical features of the flow, such as total flow duration, total forward packets, average inter-packet arrival time, maximum packet length, and others.

\footnotetext{
${ }^{1}$ End-to-end generation of CSE-CIC-IDS2018 is not reproducible; however, our partial analysis shows that many problems were inherited, including tails
} 
- Machine learning benchmarks. Since the dataset is meant for design and optimization of data-driven network security solutions, the final stage of its creation is performing ML benchmarks. Benchmarking includes designing, training and testing one or more ML models on the constructed dataset for a lab evaluation of network intrusion detection. This stage is meant to confirm that the dataset and its features are meaningful and appropriate for MLbased NIDS, and optionally to compare performance of various algorithms.

Understanding the underlying mechanisms of dataset generation enables researchers and practitioners to properly assess relevance of the data to the problem at hand, foresee potential pitfalls of applying machine learning to these data, and essentially set correct expectations. Dataset documentation is crucial in facilitating this analysis, and in case of CICIDS2017 we use several sources of documentation: (i) the original paper [5], (ii) the website page describing the dataset and its features [14], and (iii) the github page of the feature extraction tool [15]. In the following, we describe our analysis process for each dataset generation stage of interest, in the order applied during our study, and report our findings.

\section{A. Flow construction}

CICIDS2017 flows are constructed from raw PCAP files using the CICFlowMeter tool [15] by the same authors, which outputs CSV files where each row corresponds to a flow, and each flow has 83 (excluding the label) features. They define a flow as an exchange of network packets in both directions belonging to the same 5-tuple - a unique set \{source IP address, destination IP address, source port, destination port, transport layer protocol\} - within a certain time period.

1) TCP "tails". We analyze the source code of CICFlowMeter to understand how each flow is recorded. A flow is started upon observing a packet that does not belong to an active flow. A flow is terminated either upon timing out, or when the network connection is closed. In this dataset the timeout value was set to 120 seconds. When the underlying network protocol is TCP, CICFlowMeter considers the network connection closed (and the corresponding flow completed) upon detecting the first packet in the flow that contains a FIN flag. We notice that this design decision violates the TCP specification [16] that stipulates that a FIN flag merely indicates that the sender is done transmitting data. A TCP connection, however, is only terminated when both sides have sent a FIN packet to each other.

The result of the misunderstanding of the TCP mechanism has rather drastic consequences: if a flow is considered terminated already after the first of the actual two FIN packets, any subsequent packets belonging to the same TCP connection will constitute their own flow. These flows mostly consist of just two leftover ACK and FIN packets and are labeled as the original flow, be that benign or an attack. We will henceforth refer to these flows as "tails", since they constitute the tail end of the underlying TCP connection. In Section IV we show that the tails are alarmingly present, making up 25.9 percent of the entire dataset and study their impact.

2) Timeout and tails. After a flow timeout, a subsequent flow with the same 5-tuple maintains the source and destination of the timed out flow, which guarantees consistency across multiple flows spanning a single (long) TCP connection. However, the presence of TCP tails, in malicious traffic specifically, breaches this logic: sometimes a tail times out, and then a new attack flow with the same 5-tuple assumes the source and destination from the tail, which are often incorrect (depending on direction of the first tail packet). This eventually leads to mislabeling the flow as benign. When we reconstruct the flows to eliminate tails, we simultaneously return correct attack labels to such flows.

3) RST omission. According to the specification [16], the RST packet is used to reset a TCP connection. We observe, however, that the CICFlowMeter tool does not consider the RST packet as a valid way to terminate a TCP flow. We fix this and other flow construction issues in the tool, so that TCP flows more accurately reflect their underlying TCP connection.

\section{B. Labeling}

The authors provide information about the IP addresses of attackers and victims as well as the time window during which each attack was executed, which should allow for reproducibility of the labelling logic.

1) Time frames. We found that the time frames reported on the dataset information page [14] are not precise, therefore we had to tune them to reproduce the labelling. We will report these details along with the new version of the dataset for future usage.

2) Sanity check. A crucial observation is that the employed labeling strategy relies entirely on a specific data collection window, labeling a traffic flow between an attacker and a victim as malicious simply on the grounds of being collected at a certain moment in time. Unfortunately, a resulting flow's content and characteristics are not verified to ensure that malicious or suspicious activity is taking place.

We found at least one potential issue with that, which affects most attack classes. All attacks, except for PortScan, rely on an established TCP connection in order to deploy malicious functionality. Within this established TCP connection, there needs to be some kind of communication in the form of a payload for the attack to take place.

However, we observe that Web Attack - Brute Force and Web Attack - XSS largely consist of flows that have no data transfer in the forward direction, which means that the attacker never actually executes an attack within those flows. Bot traffic also contains a large number of failed or empty TCP connections, which do not contain meaningful botnet traffic and thus should not be labelled as such. Moreover, when a DoS attack brings a web server down, making it unable to properly respond to new incoming connection requests, these numerous TCP connections without a HTTP payload are still labeled as an instance of a HTTP-based DoS attack. 
We conclude that labeling these examples as attacks is inappropriate for the type of a flow-based NIDS which analyzes each flow in isolation from another and cannot judge whether an observed failed or empty TCP connection is a part of a larger ongoing malicious campaign. That is, CICIDS2017 flows that are generated by a connection initiated by an attack simulation tool but do not contain any forward data transfer are by nature benign, and we relabel them as such (IV-B).

\section{Attack simulation}

Most attacks are generated by executing an automated tool from one or more hosts in the attacker network, while other attacks are executed by Python scripts written by the authors. Some attack categories (such as Bot, Infiltration) assume that the victim host is compromised, after which the victim will be initiating connections with the attacker.

1) DoS Hulk misimplementation. Our analysis reveals that the DoS Hulk tool used in this dataset simulation is deprecated and should not be used to execute a DoS attack. The DoS Hulk tool aims to cause the target web-server to keep a connection open during 110-120 seconds. However, instead of setting the Connection header field in the HTTP request to Keep-Alive, this implementation sets it to Close. As a result, the web server sets the FIN flag on the outgoing HTTP response packet, initiating to close the connection. As this renders the DoS Hulk attack ineffective, we do not believe this attack class in CICIDS2017 can be used as intended.

2) Countermeasures. While the authors have published more metadata about the data collection infrastructure than is usually seen for NIDS datasets, there is still insufficient transparency about the countermeasures put in place on victim hosts. For the Apache web server, our analysis suggests that the authors ran the Apache web server on default settings, meaning that the Keep-Alive Timeout was set to 5 seconds. As a result, the DoS GoldenEye attack (with a strategy similar to DoS Hulk) was rendered much less effective. This design choice further hinders the diversity property.

3) Diversity. The authors of the dataset ensure diversity in benign behaviour and include a wide range of attacks. However, diversity of data within each attack category is not openly discussed. The authors did not specify the parameters that they used when running the attack tools, and the code for implemented attacks is not published. We are not aware whether the attack tools were used with a certain fixed configuration or with varying parameters. This affects how representative each attack class is of the overall attack strategy. A supervised NIDS trained on attack traces that were simulated using one configuration of the attack tool may not generalize to other configurations, making NIDS behaviour unpredictable on slightly differing attack implementations.

Moreover, as our evaluation shows, some attack tools hardcode packet lengths, drawing too much attention to this feature during training. For the sake of diversity within a single attack category, it is worth to consider combining several tools and their configurations for attack simulation.

\section{Feature extraction}

The dataset uses flow-level statistical features as a traffic representation. These features are calculated in the CICFlowMeter feature extraction tool.

1) Note of caution. The original CSV files still contain attributes such as Flow ID, Source IP, Destination IP, Source Port, Destination Port, and Timestamp of the flow. Whereas using the destination port as a NIDS feature is up for debate, the other attributes should be excluded from the feature representation during training together with the label. This should prevent a flow-based NIDS from associating a certain timestamp or some host-specific information with a certain class, without actually learning the underlying problem.

2) Absolute timestamp. When reproducing feature extraction, we notice an implementation error in the public version of CICFlowMeter that leads to encoding the absolute timestamp in statistics that describes how long a flow was Active or Idle. As predicted above, a ML-based NIDS will seamlessly memorize the timestamp of generation of different attacks when trained on these 8 features, ending up with near-perfect performance. We were able to detect the problem when analyzing the scores of importance of various features in prediction, which we now recommend as a sanity check for any featurebased classifier. Fortunately, the error was not present when CICIDS2017 and CSE-CIC-IDS2018 were created; however, any new generated dataset will be affected.

3) Minor fixes. The older version of CICFlowMeter used to generate the dataset seems to have had a couple of minor errors such as a duplicate Fwd Header Length feature and incorrect TCP flag count, which are present in CICIDS2017. In the new version of CICFlowMeter, there is still an issue of incorrectly calculated Fwd PSH Flags and Bwd PSH Flags counts, affecting also the new CSE-CIC-IDS2018 dataset.

\section{E. Machine learning benchmarks}

The authors of CICIDS2017 evaluate standard ML classifiers [5]: Random Forest (RF), Multi-Layer Perceptron (MLP), K-Nearest Neighbors, etc. Apart from execution speed for training and testing, they report aggregated performance metrics for the whole test set, based on the total number of true positives (TP), false positives (FP) and false negatives (FN):

Precision: the ratio of correctly detected attacks to all traces classified as malicious $T P /(T P+F P)$;

Recall: the ratio of correctly detected attacks to all actually malicious traces $T P /(T P+F N)$;

F1-score: the harmonic mean of precision and recall.

We list a number of issues with the conducted benchmarks, which affect reliability and reproducibility of reported performance estimates. First, the lack of per-class performance measurement, such as a confusion matrix or per-class F1-score, does not allow to fully assess utility of CICIDS2017 for NIDS. Providing only aggregated precision, recall and F1-score for a given highly imbalanced dataset obscures effectiveness of the ML algorithms for different attack classes. Our evaluation shows that a ML-based NIDS fails on numerous flows of some of the attacks, encouraging deeper investigation. We 
provide revised ML benchmarks according to the suggested methodology in the next section.

\section{Evaluation}

In this section we evaluate a Random Forest classifier on the original and corrected datasets and analyze the difference. Through these benchmarks we also empirically investigate the impact of tails and mislabelled traces on ML-based NIDS, and illustrate why it is so challenging to detect such errors.

\section{A. Regenerating the dataset}

We analyze the impact of improvements suggested in Section III by applying dataset transformations in stages. First we reconstruct the Original dataset with the original CICFlowMeter tool and label it in such a way that it matches the public version as closely as possible. Next, we create an Intermediate version, where the tails problem is eliminated and the flows are reconstructed and relabeled. Finally, we apply the payload filter to relabel the non-malicious flows and generate the Final version of the dataset. Table I reports on the number of traces per category for each transformation step.

1) Intermediate: fixing tails. We correct flow construction errors (III-A) by fixing CICFlowMeter so that the tails are systematically reunited with the "head" parts of the underlying TCP connections. Subsequently, we see a sharp decrease in flow counts across most classes in the second column of Table I. Classes where TCP connections most often finish with FIN packets see the biggest change. In some classes we also see an increase of the number of flows. This happens due to issues described in III-A2 and III-A3.

2) Final: payload filter. Following our conclusion in III-B2, we apply the payload filter to all classes except for Benign and PortScan. This filter ensures that attack classes predominantly contain flows that actually exhibit malicious activity. We notice sharp drops in several categories (Table I).

Web Attacks: Brute Force and XSS. Manual inspection of these attacks showed that the large majority of their flows do not contain any packet data. The attacks are largely executed through a select few flows, which each of them containing many brute force or XSS attempts.

Bot. Manual inspection showed that when the infected victims attempt to connect to the Botnet master, they are rejected in 2 out of 3 cases. These rejected connections carry no meaningful malicious content, and were thus relabeled.

DoS: Slowhttptest and Slowloris. With both attacks, our inspection reveals a large amount of failed TCP connections. Unlike with Bot, this can be explained by the attack strategy itself, which appears to have succeeded at bringing the victim server down, at which point it is no longer able to respond to incoming connection requests.

\section{B. Experimental results}

We perform ML benchmarks on the three dataset versions following evaluation methodology introduced in Section III-E and report results of Random Forest - the classifier with the best average F1-Score - in Table II. While the aggregated

\begin{tabular}{|c|c|c|c|}
\hline Label & Original & $\begin{array}{c}\text { Intermediate } \\
\text { (tails fixed) }\end{array}$ & $\begin{array}{c}\text { Final } \\
\text { (payload filter) }\end{array}$ \\
\hline BENIGN & 1814861 & 1823964 & 2271326 \\
\hline FTP-Patator & 7934 & 3984 & 3973 \\
\hline SSH-Patator & 5898 & 2988 & 2980 \\
\hline DoS GoldenEye & 10293 & 7647 & 7567 \\
\hline DoS Hulk & 230124 & 159048 & 158469 \\
\hline DoS Slowhttptest & 5499 & 5109 & 1742 \\
\hline DoS slowloris & 5791 & 5707 & 4001 \\
\hline Heartbleed & 11 & 11 & 11 \\
\hline Web Brute Force & 1507 & 1365 & 151 \\
\hline Web Attack XSS & 652 & 679 & 27 \\
\hline Web Attack SQL & 21 & 12 & 32 \\
\hline Infiltration & 36 & 48 & 738 \\
\hline Bot & 1956 & 2208 & 159023 \\
\hline PortScan & 158842 & 159023 & 95123 \\
\hline DDoS & 128022 & 95123 & \\
\hline
\end{tabular}

TABLE I

STRUCTURES OF THE THREE VERSIONS OF THE DATASET, WITH THE MOST PROMINENT CHANGES IN FLOW COUNTS MARKED WITH YELLOW.

performance metrics at the bottom of the table show no difference, the applied transformations have significantly affected NIDS performance for various categories. This illustrates why analyzing the imbalanced dataset at the level of aggregated ML metrics is inadequate.

1) Tails impact. We see that removing tails marginally improves performance for some classes, but also gives a performance decrease for other classes. Note that Web Attack - SQL Injection ends up with 12 traces in total, and all 3 test traces are mispredicted as Benign. This may be due to the attack being hardly distinguishable from benign traces and poorly represented in the training data. For other classes where performance worsened, the tails in the Original dataset are more likely to be classified correctly than normal attack flows. This points to the model overfitting on the tail instances, memorizing their features instead of learning actual attack patterns. The model will be able to overfit to such data only if (1) tails of different attack classes can be differentiated, and (2) tails can be distinguished from regular flows of the same class. To assess whether these conditions hold, we apply extensive feature importance measurements for the RF classifier. We omit the details due to space constraints and present high-level observations.

Tail features indeed have widely varying distributions across different classes, and most importantly, in all cases these features do not have any semantic connection with the intended malicious activity, but rather stem from the specifics of the used attack tools. We find that the model overfits through shortcut learning: it uses four prominent but largely irrelevant features to crisply differentiate between tails of different classes (Fwd Header Len, Total Fwd Pkt, Min Seg Size Fwd, and Init Win bytes $F w d$ ), and mainly two features (SYN Flag Count, Bwd Pkt Len Mean) to separate tails from regular flows.

2) Payload filter impact. The final transformation through the targeted payload filter triggers a large change towards much higher performance on Brute Force, XSS and Bot attacks. This indicates that Bot flows without an established TCP connection were a source of confusion for the RF classifier. Our experiments revealed that the presence of RST packets 


\begin{tabular}{|c|c|c|c|c|c|c|c|c|c|}
\hline & \multicolumn{3}{|c|}{ Original } & \multicolumn{3}{|c|}{ Intermediate } & \multicolumn{3}{|c|}{ Final } \\
\hline Label & Pr & $\operatorname{Re}$ & F1 & $\mathbf{P r}$ & $\mathbf{R e}$ & F1 & Pr & $\mathbf{R e}$ & F1 \\
\hline BENIGN & 0.99 & 0.99 & 0.99 & 0.99 & 0.99 & 0.99 & 0.99 & 0.99 & 0.99 \\
\hline FTP-Patator & 1.0 & 0.99 & 0.99 & 1.0 & 1.0 & 1.0 & 1.0 & 1.0 & 1.0 \\
\hline SSH-Patator & 1.0 & 0.99 & 0.99 & 1.0 & 1.0 & 1.0 & 1.0 & 0.99 & 0.99 \\
\hline DoS GoldenEye & 0.99 & 0.99 & 0.99 & 0.99 & 0.99 & 0.99 & 1.0 & 0.99 & 0.99 \\
\hline DoS Hulk & 0.99 & 0.99 & 0.99 & 0.99 & 0.99 & 0.99 & 1.0 & 0.99 & 0.99 \\
\hline DoS Slowhttptest & 0.99 & 0.99 & 0.99 & 0.99 & 0.99 & 0.99 & 0.99 & 0.99 & 0.99 \\
\hline DoS slowloris & 1.0 & 0.99 & 0.99 & 0.99 & 0.99 & 0.99 & 1.0 & 1.0 & 1.0 \\
\hline Heartbleed & 1.0 & 1.0 & 1.0 & 1.0 & 1.0 & 1.0 & 1.0 & 1.0 & 1.0 \\
\hline Web Brute Force & 0.71 & 0.94 & 0.81 & 0.68 & 0.89 & 0.77 & 1.0 & 0.87 & 0.93 \\
\hline Web Attack XSS & 0.67 & 0.11 & 0.19 & 0.46 & 0.15 & 0.23 & 1.0 & 0.43 & 0.60 \\
\hline Web Attack SQL & 1.0 & 0.20 & 0.33 & 0.0 & 0.0 & 0.0 & 0.0 & 0.0 & 0.0 \\
\hline Infiltration & 1.0 & 0.78 & 0.88 & 1.0 & 0.83 & 0.91 & 1.0 & 0.75 & 0.86 \\
\hline Bot & 0.87 & 0.50 & 0.64 & 0.80 & 0.39 & 0.53 & 1.0 & 0.99 & 0.99 \\
\hline PortScan & 0.99 & 0.99 & 0.99 & 0.96 & 0.97 & 0.97 & 0.96 & 0.97 & 0.97 \\
\hline DDoS & 0.99 & 0.99 & 0.99 & 1.0 & 1.0 & 1.0 & 1.0 & 1.0 & 1.0 \\
\hline Accuracy & & 0.99 & & & 0.99 & & & 0.99 & \\
\hline Weighted Avg & 0.99 & 0.99 & 0.99 & 0.99 & 0.99 & 0.99 & 0.99 & 0.99 & 0.99 \\
\hline
\end{tabular}

TABLE II

RANDOM FOREST AVG PERFORMANCE METRICS (TEST SET SIZE 25\%).

was a learned shortcut for Bot traffic in the Original dataset, which means that the classifier learned to recognise failed TCP connections instead of actual Botnet traffic. In the Final dataset with near-perfect benchmark results the absence of RST was an important Bot traffic feature.

We also see no significant change in performance for DoS Slowhttptest, despite the large (almost 70\%) decrease in total number of flows. However, through additional feature importance analysis we see that the model starts to learn actually relevant features, such as Inter-packet arrival time. Given that a DoS Slowhttptest attack sends intermittent fragmented HTTP packets, this feature is a strong indicator of this type of attack, and is something a human operator would look for as well.

The experiments further confirm that aggregated ML metrics computed on a large imbalanced multi-class dataset are inadequate to judge the utility of a dataset for NIDS. Class-based metrics, feature importance analysis and manual investigation of mispredictions are crucial in performance evaluation and will aid in bringing attention to suspicious data points and correlations that would otherwise have gone unnoticed.

\section{CONCLUSION}

In this paper we revised the generation process of the widely adopted CICIDS2017 dataset. Throughout analyzing traffic generation, attack simulation, flow construction, feature extraction, labeling and benchmarking stages of data collection, we discovered a series of issues that violate some of the established properties of a high quality NIDS dataset. The misimplementation of the DoS Hulk attack, the misunderstanding of the TCP protocol in flow construction and errors in feature extraction violate the requirement for correct implementation of network traffic. Notwithstanding the great effort by the CICIDS2017 team to provide documentation and metadata on the infrastructure and data collection, we still observed a lack of documentation concerning flow construction, countermeasures on victim hosts, and parameters of attacks. Moreover, an overly general labelling strategy without additional validation of malicious traces caused a significant number of flows to be mislabelled. We correct these issues and regenerate and relabel the new version of CICIDS2017 ${ }^{2}$ based on the original PCAPs. Machine learning benchmarks and feature importance analysis indicate improved validity and utility of the dataset.

Our analysis provides more insight into the process of dataset creation, which not only allows one to make informed decision when selecting the data for lab evaluations, but also - crucially - sets correct expectations and overall contributes to designing better security solutions. We believe our findings and recommendations will aid researchers and practitioners in the pursuit of better datasets and evaluation approaches.

\section{REFERENCES}

[1] H. Hindy, D. Brosset, E. Bayne, A. K. Seeam, C. Tachtatzis, R. Atkinson, and X. Bellekens, "A taxonomy of network threats and the effect of current datasets on intrusion detection systems," IEEE Access, vol. 8, pp. $104650-104675,2020$.

[2] I. Sharafaldin, A. Gharib, A. H. Lashkari, and A. A. Ghorbani, "Towards a reliable intrusion detection benchmark dataset," Software Networking, vol. 2018, no. 1, pp. 177-200, 2018.

[3] E. K. Viegas, A. O. Santin, and L. S. Oliveira, "Toward a reliable anomaly-based intrusion detection in real-world environments," Computer Networks, vol. 127, pp. 200-216, 2017.

[4] M. Ring, S. Wunderlich, D. Scheuring, D. Landes, and A. Hotho, "A survey of network-based intrusion detection data sets," Computers \& Security, vol. 86, pp. 147-167, 2019.

[5] I. Sharafaldin, A. H. Lashkari, and A. A. Ghorbani, "Toward generating a new intrusion detection dataset and intrusion traffic characterization." in ICISSP, 2018, pp. 108-116.

[6] L. Leichtnam, E. Totel, N. Prigent, and L. Mé, "Sec2graph: Network attack detection based on novelty detection on graph structured data," in International Conference on Detection of Intrusions and Malware, and Vulnerability Assessment. Springer, 2020, pp. 238-258.

[7] A. Rosay, F. Carlier, and P. Leroux, "Mlp4nids: An efficient mlp-based network intrusion detection for cicids2017 dataset," in International Conference on Machine Learning for Networking. Springer, 2019, pp. 240-254.

[8] D. Stiawan, M. Y. B. Idris, A. M. Bamhdi, R. Budiarto et al., "Cicids2017 dataset feature analysis with information gain for anomaly detection," IEEE Access, vol. 8, pp. 132911-132921, 2020.

[9] J. L. Leevy and T. M. Khoshgoftaar, "A survey and analysis of intrusion detection models based on cse-cic-ids2018 big data," Journal of Big Data, vol. 7, no. 1, pp. 1-19, 2020.

[10] A. Gharib, I. Sharafaldin, A. H. Lashkari, and A. A. Ghorbani, "An evaluation framework for intrusion detection dataset," in 2016 International Conference on Information Science and Security (ICISS). IEEE, 2016, pp. $1-6$.

[11] G. Apruzzese, M. Colajanni, and M. Marchetti, "Evaluating the effectiveness of adversarial attacks against botnet detectors," in 2019 IEEE 18th International Symposium on Network Computing and Applications (NCA). IEEE, 2019, pp. 1-8.

[12] I. Sharafaldin, A. H. Lashkari, and A. A. Ghorbani, "A detailed analysis of the cicids2017 data set," in International Conference on Information Systems Security and Privacy. Springer, 2018, pp. 172-188.

[13] A. Abdullah ALFRHAN, R. Hamad ALHUSAIN, and R. Ulah Khan, "Smote: Class imbalance problem in intrusion detection system," in 2020 International Conference on Computing and Information Technology (ICCIT-1441), 2020, pp. 1-5.

[14] C. I. for Cybersecurity, "Intrusion detection evaluation dataset (cicids2017)," https://www.unb.ca/cic/datasets/ids-2017.html, 2017, accessed: 2021-01-22.

[15] "Cicflowmeter tool," https://www.unb.ca/cic/research/applications.html, accessed: 2021-01-09.

[16] J. Postel et al., "Rfc 793: Transmission control protocol specification," 1981.

\footnotetext{
${ }^{2}$ We reported our findings to the dataset authors and will assist them in correcting the feature extraction tool. Upon publication, we will open source the analysis code and the final dataset.
} 